\title{
Permanent Closed-Loop Operation as a Measure for Improving Power Supply Reliability in a Rural Medium Voltage Distribution Network
}

\author{
G. Štumbrger ${ }^{1}$, M. Rošer ${ }^{2}$ M. Pintarič, and B. Polajžer ${ }^{1}$ \\ ${ }^{1}$ Faculty of Electrical Engineering and Computer Science \\ University of Maribor \\ Koroška cesta 46, 2000 Maribor (Slovenia) \\ Phone/Fax number: +386 22207075 ,
}

e-mail: gorazd.stumberger@um.si, matej.pintaric1@um.si, bostjan.polajzer@um.si

${ }^{2}$ Elektro Celja d.d.

Vrunčeva 3 a, 3000 Celje (Slovania)

e-mail: miran.roser@elektor-celie.si

\begin{abstract}
Power supply reliability is one of the critical elements of power quality. Its improvement in medium voltage distribution systems in urban areas is typically achieved by cabling. Unfortunately, this approach is often too expensive to be applied in rural areas. One of the possible alternative solutions that can be used in rural medium voltage distribution networks is permanent closed-loop operation. It requires the introduction of additional reclosers with properly parametrized protection relays enabling directional protection. This paper focuses on the case study performed in the $20 \mathrm{kV}$ rural distribution network of Elektro Celje d.d.. Two feeders, where the reliability of the power supply was low, were analyzed. Results of techno-economical evaluation eliminated cabling as a viable solution. The permanent closed-loop operation was selected and implemented after proper placement and parameterization of relays with directional protection functions. The results of two years operation in permanent closedloop arrangement of feeders show substantial improvement in the reliability of power supply.
\end{abstract}

Key words. rural medium voltage distribution network, permanent closed-loop operation, power supply reliability.

\section{Introduction}

Permanent operation with feeders in the closed-loop arrangement is a rarity in medium voltage distribution networks, although the idea is not new [1]. The feasibility study regarding the transition from permanent open-loop to the permanent closed-loop operation of feeders is given in [2]. The reduction in line losses and harmonic distortion that can be achieved in this way is discussed in [3], [4]. The load flow control and voltage profile-related problems are discussed in [5] and [6].

This paper focuses on improving power supply reliability in a rural $20 \mathrm{kV}$ distribution network that supplies a ski resort Slovenia. The power supply reliability was low and must be improved, while cabling of the entire feeder was related to high costs. After performing analysis of the discussed distribution network shown in Fig. 1, where existing switching devices are marked with triangles, a decision was made to replace some of the existing switching devices containing directional protection relay functionalities and introduce some additional switching devices. A schematic presentation of the discussed distribution network with equipped relays (R) is shown in Fig. 2. From this presentation, it is clear that some relays are directional.

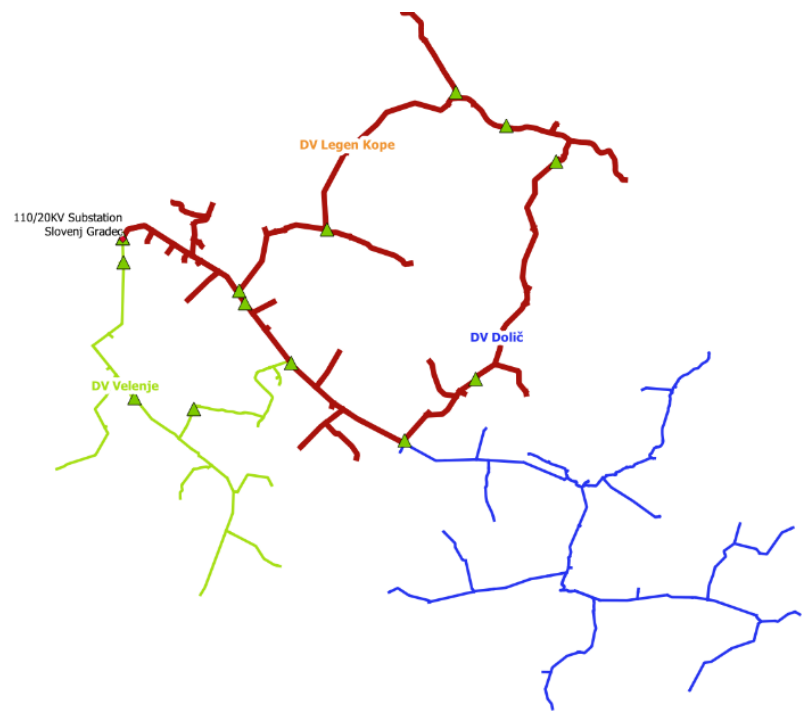

Fig.1. Discussed $20 \mathrm{kV}$ network (tringles mark positions of switching devices)

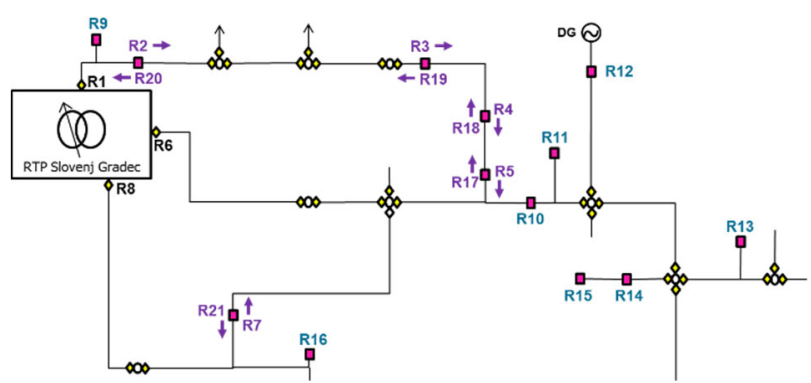

Fig.2. Discussed $20 \mathrm{kV}$ network equipped with switching devices with protection relays $(\mathrm{R})$

The results of the performed techno-economic analysis focused on the feeders DV Legen Kope (relays R1, R2, 
R3, R19, and R20) and DV Dolič (Relays R4, R5, R6, R17, and R18) supplying the ski resort Kope, showed, that this solution enables a substantial improvement in the power supply reliability at approximately $20 \%$ of the costs for cabling.

\section{Protection system setting}

In order to provide proper operation of the protection system suitable for permanent closed-loop operation of the feeders DV Legen Kope and DV Dolič, proper selectivity and sensitivity of all relay pairs was crucial.

The same procedure for optimisation of timing coordination was proposed for the over current (OC) and for the earth fault (EF) protections. Inverse IEC curves were used [7-9], where the operating time $t$ is calculated by:

$$
t=T D S \frac{A}{\left(\frac{I}{I_{\mathrm{pu}}}\right)^{B}-1}+T_{\text {add }}
$$

where $I$ denotes the rms value of a current measured by a relay. Relay setting are denoted as:

$$
\begin{array}{lll}
\text { - } & I_{\mathrm{pu}} & \text { pick-up current setting, } \\
\text { - } & T D S & \text { time delay setting, } \\
\text { - } & T_{\text {add }} & \text { additional time delay, } \\
& A, B & \text { constants according to curve type (Table }
\end{array}
$$

I).

Table I. - Constants for IEC inverse curves

\begin{tabular}{|l||l|l|}
\hline Inverse type & $A[\mathrm{~s}]$ & $B$ \\
\hline \hline Extreme & 80 & 2 \\
\hline
\end{tabular}

Selectivity is achieved by timing coordination, where the relay intended to operate (primary relay) operates faster than other relays (back-up relays). Definition of a coordination time interval $(C T I)$ is demonstrated in Fig. 3 for a fundamental relay pair consisting of a single primary and back-up relay, where $I_{\mathrm{SC}}$ is value of the current corresponding to the fault location at primary relay point. In order to achieve selectivity over an entire range of currents, the following conditions must be fulfilled:

$$
t_{\mathrm{b}}-t_{\mathrm{p}}>C T I \quad \text { and } \quad t_{\mathrm{pu}, \mathrm{b}}>t_{\mathrm{pu}, \mathrm{p}}
$$

where indices $\mathrm{p}$ and $\mathrm{b}$ stand for a primary and back-up relay, respectively.

a)

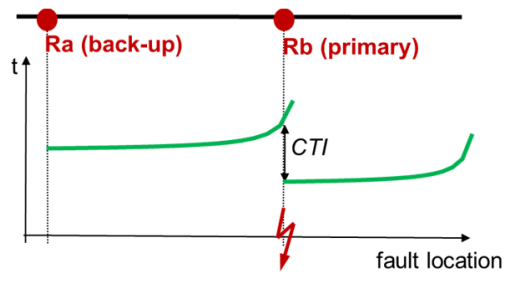

b)

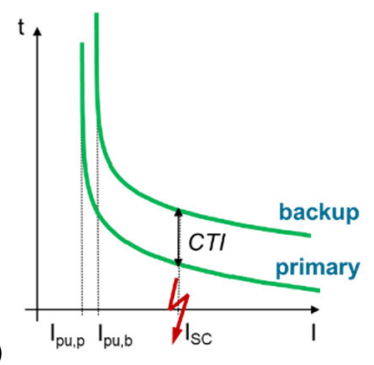

Fig.3. Definition of CTI for a relay pair: operating time vs. fault location (a) and current vs. operating time (b)

The relay pairs (RP) for the network configuration shown in Fig.2. are defined in Table II.

Table II. - Constants for IEC inverse curves

\begin{tabular}{|l||l|l|}
\hline RP no. & Primary rel. no. & Backup rel. no. \\
\hline \hline 1 & 2 & $1 \bullet$ \\
\hline 2 & 3 & 2 \\
\hline 3 & 4 & 3 \\
\hline 4 & 5 & 4 \\
\hline 5 & 17 & $6 \bullet$ \\
\hline 6 & 18 & 17 \\
\hline 7 & 19 & 18 \\
\hline 8 & 20 & 19 \\
\hline 9 & 7 & $8 \bullet$ \\
\hline 10 & 9 & $1 \bullet$ \\
\hline 11 & 9 & 20 \\
\hline 12 & 10 & 5 \\
\hline 13 & 10 & $6 \bullet$ \\
\hline 14 & 11 & 10 \\
\hline 15 & 12 & 10 \\
\hline 16 & 13 & 10 \\
\hline 17 & 14 & 10 \\
\hline 18 & 15 & 14 \\
\hline 19 & 16 & $8 \bullet$ \\
\hline & & \\
\hline
\end{tabular}

The time delay and pick-up current settings of all relays were determined in a Differential Evolution (DE) [10] based optimization procedure, where $T_{\text {add }}$ was set to 0 . The applied objective function is given by (3):

$$
\boldsymbol{T}_{\mathrm{opr}}=\sum_{\boldsymbol{r}=\mathbf{1}}^{\boldsymbol{N}_{\mathrm{RP}}}\left(\boldsymbol{t}_{\mathrm{p}, \mathrm{r}}+\boldsymbol{t}_{\mathrm{b}, \mathrm{r}}\right)+\boldsymbol{p}
$$

where index $\mathrm{r}$ stands for the relay pair, $N_{\mathrm{RP}}$ denotes the number of all relay pairs, while $p$ is the penalty. More details will be provided in the full paper.

The results presented in this paper were obtained for the protection system realization without the exchange of GOOSE messages. More details about the protection system realization are provided in [11], emphasizing the exchange of GOOSE messages.

\section{Results}

The closed-loop operation's performance with the proposed protection scheme is summarized in Table II, where the time, date, and location of the fault that appeared between individual relays (R) marked in Fig. 2 are given. The results show how many customers (Cust.) and transformers (Transf.) $20 \mathrm{kV} / 0.4 \mathrm{kV}$ were without supply in the cases of faults when the proposed protection scheme, that fully supports permanent closed-loop operation and disconnects only the faulted line sections between relays were used. Moreover, the results also show how many customers and transformers $20 \mathrm{kV} / 0.4 \mathrm{kV}$ would be without supply considering the same faults with the protection scheme used in the abandoned radial arrangement of feeders. The statistic related to the three events presented in Table III is shown in Tables IV. 
Table III. - Fault events in the first half of 2019 in the discussed distribution network

\begin{tabular}{|c|c|c|c|c|c|}
\hline \multirow{3}{*}{$\begin{array}{c}\text { Time of } \\
\text { event }\end{array}$} & \multirow{3}{*}{$\begin{array}{l}\text { Location } \\
\text { between }\end{array}$} & \multicolumn{4}{|c|}{ Disconnected } \\
\hline & & \multicolumn{2}{|c|}{$\begin{array}{c}\text { Loop } \\
\text { configuration }\end{array}$} & \multicolumn{2}{|c|}{$\begin{array}{c}\text { Radial } \\
\text { configuration }\end{array}$} \\
\hline & & Cust. & Transf. & Cust. & Transf. \\
\hline $\begin{array}{l}25.3 .2019 \\
\text { at } 19: 00\end{array}$ & $\begin{array}{l}\text { (R4, } \\
\text { R18) - } \\
\text { (R3, } \\
\text { R19) }\end{array}$ & 49 & 4 & 2124 & 80 \\
\hline $\begin{array}{l}26.04 .2019 \\
\text { at } 16: 53\end{array}$ & $\begin{array}{l}\text { R4, } \\
\text { R18) - } \\
(\mathrm{R} 3, \mathrm{R} 19)\end{array}$ & 49 & 4 & 2124 & 80 \\
\hline $\begin{array}{l}20.5 .2019 \\
\text { at } 07: 52\end{array}$ & $\begin{array}{l}(\mathrm{R} 6)- \\
\text { (R10)- } \\
(\mathrm{R} 5, \mathrm{R} 17)\end{array}$ & 1966 & 75 & 2588 & 103 \\
\hline
\end{tabular}

Table IV. - Statistics of events presented in Table III

\begin{tabular}{|c|c|c|c|c|}
\hline \multirow{2}{*}{ Event } & \multicolumn{2}{|c|}{ Interrupted customers } & \multicolumn{2}{|c|}{$\begin{array}{c}\text { Duration of interruption } \\
\text { (min) }\end{array}$} \\
\cline { 2 - 5 } & $\begin{array}{c}\text { Closed- } \\
\text { loop }\end{array}$ & Radial & $\begin{array}{c}\text { Closed- } \\
\text { loop }\end{array}$ & Radial \\
\hline 1 & 49 & 2124 & 23 & - \\
\hline 2 & 49 & 2124 & 60 & - \\
\hline 3 & 1966 & 2588 & 356 & - \\
\hline
\end{tabular}

Considering definitions for SAIFI (System Average Interruption Frequency Index) and SAIDI (System Average Interruption Duration Index)

$S A I F I=\frac{\text { total number of interruptions for a group of customers }}{\text { number of all customers }}$

SAIDI $=\frac{\text { total duration of interruptions for a group of customers }}{\text { number of all customers }}$

the results presented in Table $\mathrm{V}$ are obtained.

Table V. - Calculated SAIFI and SAIDI (CL=closed-loop, $\mathrm{Rad}=$ radial)

\begin{tabular}{|c|c|c|c|c|c|c|}
\hline \multirow{2}{*}{ Event } & \multicolumn{3}{|c|}{ SAIFI } & \multicolumn{3}{c|}{ SAIDI (min) } \\
\cline { 2 - 7 } & CL & Rad. & $\begin{array}{c}\text { Change } \\
(\%)\end{array}$ & CL & Rad. & $\begin{array}{c}\text { Change } \\
(\%)\end{array}$ \\
\hline 1 & 0.023 & 1 & $\mathbf{9 8}$ & 0.53 & 23 & $\mathbf{9 8}$ \\
\hline 2 & 0.023 & 1 & $\mathbf{9 8}$ & 1.38 & 60 & $\mathbf{9 8}$ \\
\hline 3 & 0.76 & 1 & $\mathbf{2 4}$ & 270.56 & 356 & $\mathbf{2 4}$ \\
\hline $1+2+3$ & 0.81 & 3 & $\mathbf{7 3}$ & 272.5 & 439 & $\mathbf{3 8}$ \\
\hline
\end{tabular}

Let us extend the interval of our observation to the last five years. Tables VI to X show the statistic of outages in the discussed network between years 2016 and 2020. The results presented in Tables VI to VIII are given for the years 2016 to 2018, before the permanent closed-loop was implemented. The results presented in Tables IX and X are provided for 2019 and 2020 after the implementation of the closed-loop operation. Based on the results presented, it is evident that the implementation of permanent closed-loop operation cannot reduce the number of events that lead to the outages. However, it can substantially reduce the duration of outages. In the given case, the permanent closedloop operation decreased the average yearly duration of outages per customer from $329 \mathrm{~min} /$ customer to 111 $\mathrm{min} /$ customer. The presented data include the outages caused by faults as well as the outages caused due to maintenance. Although the users are without a power supply, the last ones are usually not included in SAIDI.
Table VI. Outages in 2016 (Out = Outage; All = All Customers; Aff $=$ Affected Customers, Dur $=$ Duration $)$

\begin{tabular}{|c|c|c|c|c|c|}
\hline Out & Aff & $\begin{array}{c}\text { Dur } \\
{[\mathrm{min}]}\end{array}$ & Aff/All & $\begin{array}{c}\text { Dur* } \\
\text { (Aff/All) }\end{array}$ & All \\
\hline 1 & 477 & 92.00 & 0.581 & 53.452 & 821 \\
\hline 2 & 184 & 1.02 & 0.224 & 0.228 & 821 \\
\hline 3 & 184 & 5.00 & 0.224 & 1.121 & 821 \\
\hline 4 & 477 & 248.00 & 0.581 & 144.088 & 821 \\
\hline 5 & 344 & 37.00 & 0.419 & 15.503 & 821 \\
\hline 6 & 821 & 0.92 & 1.000 & 0.917 & 821 \\
\hline 7 & 821 & 0.05 & 1.000 & 0.050 & 821 \\
\hline 8 & 821 & 1.00 & 1.000 & 1.000 & 821 \\
\hline 9 & 177 & 143.00 & 0.216 & 30.829 & 821 \\
\hline 10 & 76 & 0.38 & 0.036 & 0.014 & 2137 \\
\hline 11 & 76 & 0.22 & 0.036 & 0.008 & 2137 \\
\hline 12 & 76 & 1.57 & 0.036 & 0.056 & 2137 \\
\hline 13 & 2137 & 1.22 & 1.000 & 1.217 & 2137 \\
\hline 14 & 1976 & 5.90 & 0.925 & 5.455 & 2137 \\
\hline 15 & 1320 & 13.42 & 0.618 & 8.287 & 2137 \\
\hline 16 & 45 & 243.68 & 0.021 & 5.131 & 2137 \\
\hline 17 & 2137 & 31.28 & 1.000 & 31.283 & 2137 \\
\hline 18 & 2137 & 39.00 & 1.000 & 39.000 & 2137 \\
\hline 19 & 76 & 133.95 & 0.036 & 4.764 & 2137 \\
\hline 20 & 23 & 299.00 & 0.011 & 3.218 & 2137 \\
\hline 21 & 2137 & 0.60 & 1.000 & 0.600 & 2137 \\
\hline 22 & 2137 & 0.78 & 1.000 & 0.783 & 2137 \\
\hline 23 & 1968 & 0.28 & 0.921 & 0.261 & 2137 \\
\hline \multicolumn{7}{|l|}{ Total [min/(customer year)] } & 347.265 & \\
\hline
\end{tabular}

Table VII. Outages in 2017 (Out = Outage; All = All

Customers; Aff = Affected Customers, Dur =Duration)

\begin{tabular}{|c|c|c|c|c|c|}
\hline Out & Aff & $\begin{array}{c}\text { Dur } \\
{[\mathrm{min}]}\end{array}$ & Aff/All & $\begin{array}{c}\text { Dur* } \\
\text { (Aff/All) }\end{array}$ & All \\
\hline 1 & 183 & 2.95 & 0.223 & 0.657 & 822 \\
\hline 2 & 183 & 1.50 & 0.223 & 0.334 & 822 \\
\hline 3 & 822 & 1.27 & 1.000 & 1.267 & 822 \\
\hline 4 & 822 & 1.25 & 1.000 & 1.250 & 822 \\
\hline 5 & 79 & 11.20 & 0.037 & 0.413 & 2140 \\
\hline 6 & 78 & 0.95 & 0.036 & 0.035 & 2140 \\
\hline 7 & 78 & 1.87 & 0.036 & 0.068 & 2140 \\
\hline 8 & 361 & 93.77 & 0.169 & 15.818 & 2140 \\
\hline 9 & 2140 & 0.45 & 1.000 & 0.450 & 2140 \\
\hline 10 & 2140 & 0.33 & 1.000 & 0.333 & 2140 \\
\hline 11 & 427 & 2.08 & 0.200 & 0.416 & 2140 \\
\hline 12 & 118 & 0.55 & 0.055 & 0.030 & 2140 \\
\hline 13 & 1257 & 10.00 & 0.587 & 5.874 & 2140 \\
\hline 14 & 24 & 56.78 & 0.011 & 0.637 & 2140 \\
\hline 15 & 118 & 66.53 & 0.055 & 3.669 & 2140 \\
\hline 16 & 88 & 235.72 & 0.041 & 9.693 & 2140 \\
\hline 17 & 78 & 86.80 & 0.036 & 3.164 & 2140 \\
\hline 18 & 78 & 14.27 & 0.036 & 0.520 & 2140 \\
\hline 19 & 2140 & 5.05 & 1.000 & 5.050 & 2140 \\
\hline 20 & 50 & 124.88 & 0.023 & 2.918 & 2140 \\
\hline 21 & 24 & 95.25 & 0.011 & 1.068 & 2140 \\
\hline 22 & 2140 & 3.65 & 1.000 & 3.650 & 2140 \\
\hline 23 & 2140 & 1.20 & 1.000 & 1.200 & 2140 \\
\hline \multicolumn{2}{|l|}{ Total [min/(customer year)] } & 58.513 & \\
\hline
\end{tabular}

\section{Conclusion}

The proposed paper deals with the permanent closed-loop operation as a measure for improving power supply reliability in rural medium voltage distribution networks. The proposed solution was implemented in the distribution network of Elektro Celje d.d., where permanent closedloop operation started in January 2019. The power supply reliability has been improved by introducing additional 
switching devices with properly parameterized relays, which disconnect only the faulty section of the loop. In this way, a substantial improvement in power supply reliability has been improved substantially. The cost of its implementation was approximately $20 \%$ of the cabling costs. Two years of operation clearly show that the applied solution did not reduce the number of outages, but it reduced their duration to $33 \%$ of the initial value (111 min vs 329 min per customer per year).

Table VIII. Outages in 2018 (Out = Outage; All = All Customers; Aff $=$ Affected Customers, Dur =Duration $)$

\begin{tabular}{|c|c|c|c|c|c|}
\hline Out & Aff & $\begin{array}{c}\text { Dur } \\
{[\mathrm{min}]}\end{array}$ & Aff/All & $\begin{array}{c}\text { Dur* } \\
\text { (Aff/All) }\end{array}$ & All \\
\hline 1 & 484 & 190,35 & 0,587 & 111,672 & 825 \\
\hline 2 & 341 & 14,48 & 0,413 & 5,986 & 825 \\
\hline 3 & 484 & 84,37 & 0,587 & 49,495 & 825 \\
\hline 4 & 825 & 8,58 & 1,000 & 8,583 & 825 \\
\hline 5 & 825 & 190,62 & 1,000 & 190,617 & 825 \\
\hline 6 & 183 & 764,28 & 0,222 & 169,532 & 825 \\
\hline 7 & 2145 & 9,90 & 1,000 & 9,900 & 2145 \\
\hline 8 & 2145 & 0,25 & 1,000 & 0,250 & 2145 \\
\hline 9 & 1425 & 0,02 & 0,664 & 0,011 & 2145 \\
\hline 10 & 2145 & 20,88 & 1,000 & 20,883 & 2145 \\
\hline 11 & 149 & 31,37 & 0,069 & 2,179 & 2145 \\
\hline 12 & 24 & 100,55 & 0,011 & 1,125 & 2145 \\
\hline 13 & 24 & 1023,52 & 0,011 & 11,452 & 2145 \\
\hline 14 & 78 & 1,63 & 0,036 & 0,059 & 2145 \\
\hline \multicolumn{7}{|c|}{ Total [min/(customer year)] } & 518.745 & \\
\hline
\end{tabular}

Table IX. Outages in 2019 (Out $=$ Outage; All = All Customers; Aff $=$ Affected Customers, Dur $=$ Duration $)$

\begin{tabular}{|c|c|c|c|c|c|}
\hline Out & Aff & $\begin{array}{c}\text { Dur } \\
{[\mathrm{min}]}\end{array}$ & Aff/All & $\begin{array}{c}\text { Dur* } \\
\text { (Aff/All) }\end{array}$ & All \\
\hline 1 & 43 & 12.48 & 0.050 & 0.630 & 852 \\
\hline 2 & 852 & 2.37 & 1.000 & 2.370 & 852 \\
\hline 3 & 293 & 0.00 & 0.344 & 0.000 & 852 \\
\hline 4 & 27 & 578.35 & 0.032 & 18.328 & 852 \\
\hline 5 & 267 & 59.10 & 0.313 & 18.521 & 852 \\
\hline 6 & 852 & 5.67 & 1.000 & 5.667 & 852 \\
\hline 7 & 1282 & 2.28 & 1.505 & 3.436 & 852 \\
\hline 8 & 792 & 0.32 & 0.930 & 0.294 & 852 \\
\hline 9 & 451 & 24.77 & 0.529 & 13.110 & 852 \\
\hline 10 & 46 & 49.35 & 0.054 & 2.664 & 852 \\
\hline 11 & 293 & 2.07 & 0.344 & 0.711 & 852 \\
\hline 12 & 50 & 23.28 & 0.023 & 0.543 & 2145 \\
\hline 13 & 24 & 187.47 & 0.011 & 2.098 & 2145 \\
\hline 14 & 50 & 60.62 & 0.023 & 1.413 & 2145 \\
\hline 15 & 26 & 71.08 & 0.012 & 0.862 & 2145 \\
\hline 16 & 138 & 60.58 & 0.064 & 3.898 & 2145 \\
\hline 17 & 26 & 163.07 & 0.012 & 1.977 & 2145 \\
\hline 18 & 91 & 129.72 & 0.042 & 5.503 & 2145 \\
\hline 19 & 26 & 256.45 & 0.012 & 3.108 & 2145 \\
\hline 20 & 1984 & 0.80 & 0.925 & 0.740 & 2145 \\
\hline 21 & 2123 & 5.95 & 0.990 & 5.889 & 2145 \\
\hline 22 & 163 & 35.63 & 0.076 & 2.708 & 2145 \\
\hline 23 & 2123 & 13.37 & 0.990 & 13.230 & 2145 \\
\hline 24 & 79 & 259.27 & 0.037 & 9.549 & 2145 \\
\hline 25 & 44 & 404.42 & 0.021 & 8.296 & 2145 \\
\hline 26 & 79 & 1.57 & 0.037 & 0.058 & 2145 \\
\hline 27 & 88 & 4.90 & 0.041 & 0.201 & 2145 \\
\hline 28 & 1959 & 5.60 & 0.913 & 5.114 & 2145 \\
\hline Total [min/(customer year)] & 130.915 & \\
\hline & & & & & \\
\hline
\end{tabular}

Table X. Outages in 2020 (Out = Outage; All = All Customers; Aff $=$ Affected Customers, Dur $=$ Duration)

\begin{tabular}{|c|c|c|c|c|c|}
\hline Out & Aff & $\begin{array}{c}\text { Dur } \\
{[\mathrm{min}]}\end{array}$ & Aff/All & $\begin{array}{c}\text { Dur* } \\
\text { (Aff/All) }\end{array}$ & All \\
\hline 1 & 24 & 1.00 & 0.029 & 0.029 & 840 \\
\hline 2 & 467 & 0.93 & 0.556 & 0.519 & 840 \\
\hline 3 & 409 & 1.73 & 0.487 & 0.844 & 840 \\
\hline 4 & 120 & 2.53 & 0.143 & 0.362 & 840 \\
\hline 5 & 119 & 2.80 & 0.142 & 0.397 & 840 \\
\hline 6 & 840 & 5.60 & 1.000 & 5.600 & 840 \\
\hline 7 & 540 & 13.88 & 0.643 & 8.925 & 840 \\
\hline 8 & 60 & 302.88 & 0.071 & 21.635 & 840 \\
\hline 9 & 24 & 57.05 & 0.029 & 1.630 & 840 \\
\hline 10 & 80 & 26.80 & 0.037 & 1.000 & 2145 \\
\hline 11 & 44 & 118.67 & 0.021 & 2.434 & 2145 \\
\hline 12 & 80 & 943.37 & 0.037 & 35.184 & 2145 \\
\hline 13 & 44 & 136.35 & 0.021 & 2.797 & 2145 \\
\hline 14 & 44 & 38.15 & 0.021 & 0.783 & 2145 \\
\hline 15 & 44 & 15.40 & 0.021 & 0.316 & 2145 \\
\hline 16 & 26 & 1.00 & 0.012 & 0.012 & 2145 \\
\hline 17 & 2096 & 2.50 & 0.977 & 2.443 & 2145 \\
\hline 18 & 110 & 12.98 & 0.051 & 0.666 & 2145 \\
\hline 19 & 26 & 0.52 & 0.012 & 0.006 & 2145 \\
\hline 20 & 140 & 44.02 & 0.065 & 2.873 & 2145 \\
\hline 21 & 166 & 29.33 & 0.077 & 2.270 & 2145 \\
\hline 22 & 26 & 110.67 & 0.012 & 1.341 & 2145 \\
\hline \multicolumn{2}{|c|}{ Total [min/(customer year)] } & 92.064 & \\
\hline
\end{tabular}

\section{Acknowledgement}

The authors thank ELES d.o.o. for financing the subproject Closed-loop operation in the scope of NEDO demonstration project, grant no. POG2017/0502, and Slovenian Research Agency (ARRS) for supporting our research, grants no. P2-0115 and J2-1742.

\section{References}

[1] W. T. Huang, T. H. Chen, G. C. Pu, Y. F. Hsu, T. Y. Guo, Assessment of Upgrading Existing Primary Feeders from Radial to Normally Closed Loop Arrangement, IEEE Transmission and Distribution Conference and Exhibition, 3, 2002.

[2] Y. Guo, Feasibility Study of Upgrading Primary Feeders from Radial and Open-Loop to Normally Closed-Loop Arrangement, IEEE Transactions on Power Systems (2004), vol. 19, no. 3, pp. $1308-1316$.

[3] W. T. Huang, S. T. Chen, Line Loss Reduction by Distribution System Upgrading from Radial to Normally ClosedLoop Arrangement, Ninth International Conference on Hybrid Intelligent Systems, Washington, USA, vol. 3, pp. 334 - 339, 2009.

[4] K. Deželak, M. Rošer, R. Škof, T. Kastelic, and G. Stumberger, The impact of feeders in closed-loop arrangement on harmonic distortion and power losses, International Conference on Renewable Energies and Power Quality (ICREPQ’13), Bilbao (Spain), March 2013.

[5] N. Okada, H. Kobayashi, K. Takigawa, M. Ichikawa, K. Kurokawa, Loop power flow control and voltage characteristics of distribution system for distributed generation including PV system, 3rd World Conference on Photovoltoic Energy Conversion, May 11-18, Osaka. Japan, pp. 2284-2287, 2003.

[6] G. Štumberger, K. Deželak, M. Rošer, R. Škof, and T. Kastelic, Medium-voltage distribution feeders in open-loop and closed-loop arrangement, International Conference on Renewable Energies and Power Quality (ICREPQ'12), Santiago de Compostela (Spain), March 2012,

[7] M. Ojaghi and R. Ghahremani, Piece-wise linear characteristic for coordinating numerical overcurrent relays, IEEE Trans. Power Del. (2017), vol. 32, no. 1, pp. 145-151. 
[8] Measuring Relays and Protection Equipment- Part 151: Functional Requirements of Over/Under Current Protection, Standard IEC 60255-151, 2009.

[9] Inverse-Time Characteristics Equations for Overcurrent Relays, IEEE, Standard C37.112-2018, 2018.

[10] K. V. Price, R. M. Storn in J. A. Lampinen, Differencial Evolution, Springer, 2005.

[11] B. Polajžer, M. Pintarič, M. Rošer, and G. Štumberger, Protection of MV Closed-Loop Distribution Networks with BiDirectional Overcurrent Relays and GOOSE Communications, IEEE Access (2019), vol. 7, pp. 165884 - 165896. 